\title{
DUST OBSCURATION EVENTS OF THE SYMBIOTIC NOVA RR Tel IN THE NEAR IR
}

\author{
D. Kotnik-Karuza, ${ }^{1}$ M. Friedjung, ${ }^{2}$ and P. A. Whit.clor $\mathrm{k}^{3}$
}

By use of ground based IR photometry, we have studied the dust obscuration events of the symbiotic nova RR Tel which is a binary composed of a cool mira and a hot white dwarf. The JHKL light curves were corrected for Mira pulsations and correlated to the AAVSO visual light curve. We compare the behaviour of the three obscurations in different wavelength bands and propose interpretations of the results.

According to the results obtained from the optical emission spectra of $\mathrm{RR}$ Tel, there is more dust toward the cool star than on the line of sight to the hot regions.

Hence, in order to obtain insight into the dust regions of this binary system, we have applied IR photometry to study its cool component region. The broad band JHKL magnitudes were observed at the SAAO in South Africa over more than 25 years.

Besides the mira pulsations with a period of 387 days, long-term variations have been observed as in most symbiotic miras. After removing the short term variations by an approximate procedure, three obscuration events in all IR bands can be clearly resolved from the smoothed curves (Figure 1). The amplitudes of variations are decreasing toward longer wavelengths. The vertical shift of the $\mathrm{J}, \mathrm{H}, \mathrm{K}, \mathrm{L}$ light curves toward lower magnitudes indicates obscuration from dust that is wavelength dependent and much weaker at $L$ than at shorter wavelengths. Looking separately for correlations between different bands during the three obscuration events, we obtained a clearly different correlation of $\mathrm{L}$ with $\mathrm{J}$ than at other wavelengths. From a more detailed statistical approach, it is obvious that the $\mathrm{J}(\mathrm{K})$ and $\mathrm{K}(\mathrm{L})$ correlations are clearly different for JD less than 2446000 than at later dates when $R R$ Tel appears to be redder at a given brightness. Assuming that dust was permanently present in the

\footnotetext{
${ }^{1}$ Physics Dept., Faculty of Philosophy, University in Rijeka, Omladinska 14, 51000 Rijeka, Croatia (kotnik@mapef. pefri.hr).

${ }^{2}$ Institut d'Astrophysique de Paris, 98bis, Boulevard Arago, 75014 Paris, France.

${ }^{3} \mathrm{SAAO}, \mathrm{PO}$ Box 9, 7935 Observatory, South Africa.
}

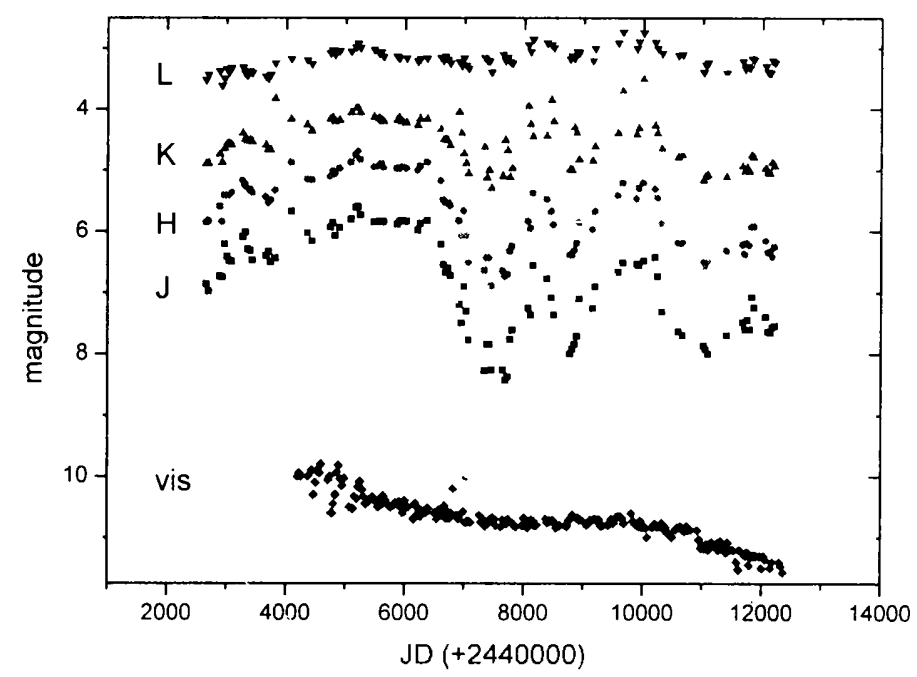

Fig. 1. JHKL light curves corrected for mira pulsations and the visual light curve over about 25 years

system, this effect could be attributed to a change in the dust properties.

Comparison with the visual light curve shows that optical variations are only approximately related to IR variations. The dust obscuration ev'nt starting about JD2450000 appears to be connected with the drop in the visual, the amplitude being stronger in $J$ than in $\mathrm{V}$ since much of the light is of nebular origin. For the same reason there is no trace of mira pulsations in the visual. The shallow merged dust fading in $\mathrm{V}$ between JD2447000 and JD2450000 most probably occurs from dust which appears distant as a mixture from different ejection events.

We are grateful to Janet Mattei and Rebecca Pollock for providing us the optical data on RR Tel.

\section{REFERENCES}

Kotnik-Karuza, D., Friedjung, M.. Fxter, K. Keenan, F P., \& Pollacco, D.L. 2003, ASP Conf. Ser., in press Mikolajewska, J., Brandi, E., Hack, W. Whitelock. P. A., Barba, R., Garcia, L., \& Marang, F. 1(4)9. MNR.ls 305,190

Whitelock, P. A. 1987, PASP, 99.573

Whitelock, P. A. 2003, ASP ('onf. S'r.. in press 